\title{
21.
}

\section{ON JACOBI'S ELLIPTIC FUNCTIONS, IN REPLY TO THE REV. B. BRONWIN; AND ON QUATERNIONS.}

[From the Philosophical Magazine, vol. xxvi. (1845), pp. 208, 211.]

The first part of this Paper is omitted, see [17]: only the Postscript on Quaternions, pp. 210, 211, is printed.

IT is pośsible to form an analogous theory with seven imaginary roots of $(-1)$ (? with $\nu=2^{n}-1$ roots when $\nu$ is a prime number). Thus if these be $i_{1}, i_{2}, i_{3}, i_{4}, i_{5}, i_{6}, i_{7}$, which group together according to the types

$$
123,145,624,653,725,734,176 \text {, }
$$

i.e. the type 123 denotes the system of equations

$$
\begin{aligned}
& i_{1} i_{2}=i_{3}, \quad i_{2} i_{3}=i_{1}, \quad i_{3} i_{1}=i_{2}, \\
& i_{2} i_{1}=-i_{3}, \quad i_{3} i_{2}=-i_{1}, \quad i_{1} i_{3}=-i_{2},
\end{aligned}
$$

\&c. We have the following expression for the product of two factors:

$$
\begin{aligned}
&\left(X_{0}+X_{1} i_{1}+\ldots X_{7} i_{7}\right)\left(X_{0}^{\prime}+X_{1}^{\prime} i_{1}+\ldots X_{7}^{\prime} i_{7}\right) \\
&= X_{0} X_{0}^{\prime}-X_{1} X_{1}^{\prime}-X_{2} X_{2}^{\prime} \ldots-X_{7} X_{7}^{\prime} \\
&+[\overline{23}+\overline{45}+\overline{76}+(01)] i_{1} \\
&+[\overline{31}+\overline{46}+\overline{57}+(02)] i_{2} \\
&+[\overline{12}+\overline{65}+\overline{47}+(03)] i_{3} \\
&+[\overline{51}+\overline{62}+\overline{47}+(04)] i_{4} \\
&+[\overline{14}+\overline{36}+\overline{72}+(05)] i_{5} \\
&+[\overline{24}+\overline{53}+\overline{17}+(06)] i_{6} \\
&+[\overline{25}+\overline{34}+\overline{61}+(07)] i_{7}
\end{aligned}
$$

where

$$
(01)=X_{0} X_{1}^{\prime}+X_{1} X_{0}^{\prime} \ldots ; \overline{12}=X_{1} X_{2}^{\prime}-X_{2} X_{1}^{\prime} \text { \&c. }
$$

and the modulus of this expression is the product of the moduli of the factors. The above system of types requires some care in writing down, and not only with respect to the combinations of the letters, but also to their order; it would be vitiated, e.g. by writing 716 instead of 176 . A theorem analogous to that which I gave before, for quaternions, is the following:-If $\Lambda=1+\lambda_{1} i_{1} \ldots+\lambda_{7} i_{7}, X=x_{1} i_{1} \ldots+x_{7} i_{7}$ : it is immediately shown that the possible part of $\Lambda^{-1} X \Lambda$ vanishes, and that the coefficients of $i_{1}, \ldots i_{7}$ are linear functions of $x_{1}, \ldots x_{7}$. The modulus of the above expression is evidently the modulus of $X$; hence "we may determine seven linear functions of $x_{1} \ldots x_{7}$, the sum of whose squares is equal to $x_{1}^{2}+\ldots+x_{7}^{2}$." The number of arbitrary quantities is however only seven, instead of twenty-one, as it should be. 